\title{
Breve reseña histórica de la inclusión en Colombia.
}

(Brief historical review of the inclusion in Colombia.)

Camargo Muñoz, Alfonso

Universidad Santo Tomás, Tunja, Colombia

Fecha recepción: 10-08-2018

Páginas 181-187

Fecha aceptación: 30-09-2018

\section{Resumen.}

En el presente artículo aborda el tema de la inclusión educativa en Colombia, partiendo, a grandes rasgos, de los lineamientos internacionales, especialmente de la UNESCO. Entidad internacional que ha promovido una serie de lineamientos para todos los países miembros, y que, como bien se sabe, influye de alguna manera en las políticas educativas de muchos países. Acto seguido, se hace especial énfasis en tres momentos de la historia educativa reciente del país: la Constitución política de 1991, la Ley general de Educación de 1994, y por este mismo año, el trabajo realizado por la denominada Misión de los sabios, conformada por educadores, pensadores y hombres de ciencias como, Gabriel García Márquez, Rodolfo LLinás, Carlos E. Vasco, M., Elkin Patarroyo, Ángela Posada, entre otros.

Palabras clave: inclusión educativa; diversidad cultural; educación; sociedad incluyente

\begin{abstract}
.
This article addresses the issue of educational inclusion in Colombia, starting, in broad strokes, from international guidelines, especially from UNESCO, an international entity that has promoted a series of guidelines for all member countries, and that, as a good it is known, influences in some way the educational policies of many countries. Then, special emphasis is placed on three moments of the country's recent educational history: the Political Constitution of 1991, the General Education Law of 1994, and for this same year, the work done by the so-called Mission of the Sages, formed by educators, thinkers and men of science such as Gabriel García Márquez, Rodolfo LLinás, Carlos E. Vasco, M., Elkin Patarroyo, Ángela Posada, among others.
\end{abstract}

Keywords: educational inclusion; cultural diversity; education; inclusive society 


\section{Introducción.}

El tratamiento que en el campo de la educación ha recibido el fenómeno de las diferencias entre los sujetos, se puede clasificar a grandes rasgos en cuatro tipos: exclusión, segregación, integración, inclusión. La exclusión se caracteriza por la actitud sistemática de prescindir de determinadas personas, por alguna circunstancia particular, ocasionándole daño a las mismas, y negándole al grupo social la participación de todos. La segregación consiste en la separación de personas en circunstancias diferenciadoras y especiales, y agrupándolas con el fin ofrecerles servicios que apenas son medianamente comparables con los recibidos por el grupo social estándar. La integración hace alusión a la vinculación de dichas personas en condiciones especiales, dentro del grupo social, en condición de igualdad, pero ignorando la necesidad del tratamiento de equidad. La inclusión, por su parte, hace referencia a la acogida de dichas personas en circunstancias particulares, pero en condiciones de equidad (Arnaiz, 1997). Según este autor (citado por Madrid Vivas y otros, 2011), la comunidad se convierte en el horizonte de comprensión de auténticos procesos educativos, y en espacio natural de experiencia inclusiva. En este trabajo nos referimos fundamentalmente a este último concepto.

\section{Los aportes de la UNESCO.}

La UNESCO ha procurado formular principios orientadores en cada una de sus conferencias sobre educación (Guerrero, R.C, 20103). En 1960, la Convención relativa a la Lucha contra las Discriminaciones en la educación, invoca la Declaración Universal de los Derechos Humanos donde se afirma el principio de que no deben establecerse discriminaciones y proclama el derecho de todos a la educación. En 1989, la Declaración de los Derechos del Niño, reconoce el derecho de todos los niños a recibir una educación sin discriminación. En 1990, la Declaración Mundial sobre Educación para Todos, promueve la idea de la educación inclusiva, mediante una educación básica para todos. En 1993, las Normas uniformes sobre la igualdad de oportunidades para las personas con discapacidad, promueve la idea de la integración en el campo de la educación. En 1994, la Declaración de Salamanca y el marco de acción para las necesidades educativas especiales, estipula que "las escuelas deben acoger a todos los niños, independientemente de sus condiciones físicas, intelectuales, sociales, emocionales, lingüísticas u otras. Deben acoger a niños discapacitados y niños bien dotados, a niños que viven en la calle y que trabajan, niños de poblaciones remotas o nómadas, niños de minorías lingüísticas, étnicas o culturales y niños de otros grupos o zonas desfavorecidos o marginados" (párrafo 3). En 1994, La educación encierra un tesoro, dirigido por Jaquees Delors, postula cuatro principios y propósitos de la educación, en el que el último de ellos aborda directamente la cuestión de la inclusión: aprender a ser, aprender a hacer, aprender a aprender, aprender a vivir juntos. En el año 2000, el Marco de Acción del Foro Mundial sobre la Educación y los Objetivos de Desarrollo del Milenio, exigen que todos los niños tengan acceso a una educación primaria obligatoria y gratuita. Meta que estaba trazada para el año 2015. Igualmente enfatizó la necesidad de vincular a los grupos marginados y a las niñas en las circunstancias diversas y 
especiales en que se encuentren. En el año 2001, la UNESCO inicia un Programa emblemático de la educación para todos (EPT) sobre el derecho a la educación de personas con discapacidades: hacia la Inclusión. En el 2006, la Asamblea General de las Naciones Unidas adopta la Convención sobre los derechos de las personas con discapacidad cuyo artículo 24 se dedica especialmente a la educación (ausencia de discriminación, igualdad de oportunidades; un sistema de educación inclusivo a todos los niveles; educación primaria inclusiva; enseñanza a lo largo de la vida, con miras a desarrollar plenamente el potencial humano y el sentido de la dignidad y la autoestima, y reforzar el respeto por los derechos humanos, las libertades fundamentales y la diversidad humana; desarrollar al máximo la personalidad, los talentos y la creatividad de las personas con discapacidad; hacer posible que las personas con discapacidad participen de manera efectiva en una sociedad libre; con el objetivo de la plena inclusión, etc.). Pero sería en el año 2008, en la conferencia, La educación inclusiva: el camino hacia el futuro, celebrada en Ginebra en torno a la inclusión educativa, que la UNESCO pone de relieve la estrecha relación que existe entre una educación inclusiva y el cumplimiento de los objetivos del milenio, y del propósito de la Naciones de una Educación para todos. Allí se recordó que el Grupo de Alto Nivel sobre la Educación para Todos, reunido en Dakar en diciembre de 2007, reafirmó que el logro de los objetivos de la EPT supone llegar a esos niños, jóvenes y adultos, especialmente las muchachas y las mujeres que hasta ahora han estado excluidos de las posibilidades de la educación básica. Concluye por otra parte que una educación de calidad, en consecuencia, es una educación inclusiva, ya que se propone velar por la participación plena de todos los educandos, con independencia de su sexo, condición económica o social, origen étnico o racial, situación geográfica, necesidades especiales de aprendizaje, edad o religión. En 2011, en la conferencia, La educación en el mundo, aborda todos los temas educativos, en clave de oportunidad para todos: extender y mejorar la protección y educación integrales de la primera infancia, proporcionar enseñanza primaria gratuita y obligatoria para todos, promover el aprendizaje y los programas de preparación para la vida activa para jóvenes y adultos, aumentar el número de adultos alfabetizados, lograr la igualdad entre los géneros y mejorar la calidad de la educación. En el 2015, la conferencia, La educación: ¿hacia un bien común mundial?, se desarrolla en clave de inclusión.

Según la UNESCO, citado por, Ramírez Valbuena (2017),

La inclusión es vista como un proceso de dirección y respuesta a la diversidad de necesidades de todos los aprendices a través de la participación en el aprendizaje. Las culturas y las comunidades deben reducir la exclusión en y desde la educación. Esto implica cambios y modificaciones en contenido, enfoques, estructuras y estrategias, con la visión común que cubre a todos los niños en un rango apropiado de edad y la convicción de que es responsabilidad del sistema regular, educar a todos los niños de la sociedad [...] La educación inclusiva como enfoque busca dirigirse a las necesidades de aprendizaje de todos los niños, jóvenes y 
adultos concentrando la atención específicamente en aquellos que son vulnerables a la marginalización y la exclusión (p. 4).

En este horizonte de comprensión, se ha de entender la inclusión primeramente como un propósito común tanto del Estado como de las comunidades locales, pero también como una manera de ser y obrar de los miembros de las comunidades educativas, que se hacen capaces de direccionar sus actuaciones de manera integral hacia la creación de espacios idóneos para una participación equitativa del aprendizaje.

\section{La inclusión a partir de la Nueva Constitución política de Colombia.}

La constitución política de Colombia de 1991 está concebida dentro de una nueva visión de país y sociedad, esto es, ya no tanto en el espíritu de la competencia y la discriminación, sino dentro del espíritu de la diversidad. Los constituyentes plasmaron el clamor de muchos ciudadanos y colectivos sociales que reclamaban el reconocimiento de la diversidad socio-cultural de los pueblos de Colombia. Una nueva etapa de la historia del país puede haber nacido entonces: todos los colombianos gozan de la misma igualdad de derechos y deberes, y el Estado debe garantizar a cada ciudadano dicha condición. La educación es un derecho de la persona y un servicio público que tiene una función social; con ella se busca el acceso al conocimiento, a la ciencia, a la técnica, y a los demás bienes y valores de la cultura ((Const. 1991, Art 67).

La ley General de Educación de 1994 procuró impulsar esta nueva visión de país, donde reclama la necesidad de que todos los colombianos se sientan incluidos en un mismo proyecto, desde la diversidad y la diferencia: de raza, se sexo, de color, de religión, de credo político, y demás elementos constitutivos del multiculturalismo colombino. Ya en su Art. 1 plantea:

De conformidad con el artículo 67 de la Constitución Política, define y desarrolla la organización y la prestación de la educación formal en sus niveles preescolar, básica (primaria y secundaria) y media, no formal e informal, dirigida a niños y jóvenes en edad escolar, a adultos, a campesinos, a grupos étnicos, a personas con limitaciones físicas, sensoriales y psíquicas, con capacidades excepcionales, y a personas que requieran rehabilitación social (Ley General de Educación, 1994).

La Misión de Sabios de 1994 dio un impulso considerable a estos intentos de comprender la nación dentro de los parámetros de la diversidad. Aunque, como han sostenido sus protagonistas, el documento construido por los "sabios", no ha dado todos sus frutos, sí pudo haber impulsado movimientos sociales y culturales que han venido reivindicando sus derechos, sobre todo, el derecho de ser reconocidos dentro de sus diferencias. He aquí su planeamiento al respecto: 
Colombia es un país pluricultural y multiétnico que puede utilizar con provecho el acceso de que dispone a los legados occidental, amerindio y afroamericano, a elementos de las sociedades modernas, premodernas y postmodernas. Esto le permitiría maximizar habilidades de diversas bases culturales para diseñar nuevos sistemas de aprendizaje e incorporar una variedad de orientaciones culturales al dominio del racionalismo científico y de la tecnología contemporánea (p. 35).

Esta nueva concepción de la inclusión en el país es el fruto de múltiples desarrollos socioculturales que tuvo sus inicios fuera de las fronteras de Colombia. Especialmente con la Declaración Universal de Derechos Humanos por parte de la ONU en 1948, las sociedades contemporáneas han procurado reivindicar los derechos de los seres humanos. Aunque unas sociedades han avanzado más que otras en este sentido, no hay duda que, en la inmensa mayoría de los pueblos, hoy se tiene mayor conciencia de la dignidad de todos los seres humanos.

En Colombia ha habido algunos avances, que, aunque muchos califican de incipientes, resultan significativos en la práctica cotidiana del quehacer educativo.

Algunos estudios pueden hablar de dichos avances (Ramírez Valbuena, 2017) especialmente en lo referente a la Necesidades Educativas Especiales (NEE), en cuanto hoy se puede constatar una mayor conciencia a nivel nacional, departamental, local y comunitario, del derecho que tienen los niños con necesidades especiales de poder acceder al aprendizaje en condiciones educativas que favorezcan su normal desarrollo.

Los proyectos de inclusión educativa de la mayor parte de las Secretarías de Educación del país, han ayudado a formar dicha conciencia inclusiva tanto en la sociedad, como en las comunidades locales y educativas. Con cierta atención a los educadores, como responsables directos de la atención escolares.

Sin embargo, la sociedad en general, y los expertos en particular, coinciden en pensar que falta mayor atención a los procesos de inclusión, tanto a nivel educativo, como a nivel social. Colombia es en general un país muy desigual. Los estratos socioeconómicos están muy marcados. Existen seis estratos, comenzando por los sectores muy pobres, que apenas pueden sobrevivir en medio de carencias básicas, hasta las clases privilegiadas que cuentan con niveles de vida solo equiparable a las clases ricas de los países más desarrollados. En este contexto de desigualdad es todavía utópico hablar de inclusión social y educativa.

Aunque, como plantea Ramírez Valbuena (2017), en el aula hoy se percibe una mayor aceptación de las diferencias, aún falta mayor concientización por parte de todos. Sin embargo, aquí se plantea un dilema de mayor complejidad aun, y es si el proceso de inclusión implica la aceptación de las diferencias socioeconómicas que han caracterizado a la sociedad colombiana, cocientes que estas diferencias 
encierran profundos obstáculos que impiden hablar de coherentemente de inclusión social y educativa.

En lo que sí todos están de acuerdo es en la necesidad de impulsar políticas educativas efectivas que hagan posibles procesos reales de inclusión.

\section{Conclusiones.}

En torno al concepto de inclusión educativa hay otros conceptos cercanos que podrían ser útiles como categorías de análisis epistemológico, pero también como clave de comprensión histórica. Estos son: exclusión, segregación, integración, inclusión. La inclusión educativa en Colombia, inspirada en todo caso en lineamientos internacionales, ha tenido importantes desarrollos a partir de la Constitución Política de 1991. Hoy existen circunstancias y factores que hacen viable un impulso a la inclusión en mejores y más justas medidas, esto es, proporcionalmente con las necesidades de la sociedad colombiana, caracterizada por la diferencia, la diversidad, el multiculturalismo. El impulso más importante en esta línea de pensamiento ha sido dado por la UNESCO, mediante las conferencias que cada año desarrolla en torno a la educación. Los documentos publicados por esta son dignos de análisis, reflexiones y aplicaciones contextualizadas. En Colombia persisten dificultades que impiden avanzar en procesos sólidos de inclusión educativa: desigualdades sociales, como, clasismo, estratificación, grandes brechas entre ricos y pobres, ausencia de oportunidades para amplios sectores de la sociedad. Pero también se percibe la ausencia de voluntad política a la hora de formular políticas educativas inclusivas. No se puede hablar con fundamento real de inclusión educativa sin hablar al mismo tiempo de inclusión social. Los esfuerzos en cuanto a la primera resultan incipientes sin el mejoramiento estructural de las condiciones sociales de todos.

\section{Referencias.}

Arnaiz, P. (1997). Integración y segregación, inclusión. N P. Arnáiz y R. De Haro (Eds). 10 años de integración en España: Análisis de la realidad y perspectivas de futuro. Actas de Científica Anual de para (AEDES). Murcia: Servicio de Publicaciones de (pp. 313-354).

Asamblea Nacional Constituyente. (1991). Constitución Política de Colombia 1991. Bogotá: Temis.

Guerrero, R.C. (2003). Principales aportaciones de las conferencias internacionales de educación de adultos de la Unesco al campo de la formación ocupacional. http://sid.usal.es/idocs/F8/ART10030/principalesaportaciones.pdf Recuperado el 1 de agosto de 2017.

Ley 115 de febrero 8 de 1994. Por la cual se expide la Ley General de Educación. Congreso de Colombia. 
Misión de Ciencia, Educación y Desarrollo (1994). Colombia: al filo de la oportunidad. Informe conjunto. Presidencia de la República et. al., Bogotá.

Madrid, V.D., Sánchez D.P., García C.D., y García G.M. (2011). De la exclusión a la inclusión: una forma de entender y atender a la diversidad funcional en las instituciones escolares. En, Educación y Diversidad, 5 (1) enero-junio (2011), ISSN: 1888-4857, pp. 23-31.

Ramírez Valbuena, W.Á. (2017). La inclusión: una historia de exclusión en el proceso de enseñanza aprendizaje. Cuadernos de Lingüística Hispánica, (30), 211230. doi: https://doi.org/10.19053/0121053X.n30.0.6195

UNESCO (2015). Conferencias sobre educación. Desde 1960 hasta 2015. Ginebra:

UNESCO (1994). Una revisión de las actividades de la UNESCO a la luz de la Declaración de Salamanca. Conferencia Mundial sobre necesidades educativas especiales: acceso y calidad. Salamanca: UNESCO. 